\title{
Studies of the Influence of Temperature on the Photocatalytic Activity of Tungsten Oxide
}

\author{
N. Derbasova ${ }^{1, a}$, V. Gavrish ${ }^{1, b}$, T. Chayka ${ }^{1, c^{*}}$ and O. Gavrish ${ }^{1, d}$ \\ ${ }^{1}$ Sevastopol State University, 33 Universitetskaya Street, Sevastopol, 299053, Russia \\ aderbasovanm@mail.ru. bvmgavrish@sevsu.ru I, 'KimChayka@yandex.ru, \\ dolga_gavrish_53@mail.ru
}

Keywords: Tungsten Oxide, Organic Pollutants, Photocatalytic Activity, Methylene Blue

\begin{abstract}
The results of photocatalytic activity of tungsten oxide in visible light are analyzed. It was shown that tungsten oxide has photocatalytic activity under the action of visible light in the photodegradation of the dye methylene blue. It was determined that the concentration of $15 \mathrm{mg} / \mathrm{L}$ provides the highest rate of photodecomposition of methylene blue. The optimum temperature for the process was determined. The process of methylene blue decomposition is most effective at $25{ }^{\circ} \mathrm{C}$.
\end{abstract}

\section{Introduction}

In recent years, considerable attention has been paid to obtaining materials with unique properties. Over the years, a large number of semiconductors have been developed and used as photocatalysts. Among various semiconductors, $\mathrm{TiO}_{2}$ is considered the best photocatalyst and is widely used to detoxify water from a number of organic pollutants [1-3]. However, photocatalysis using titanium dioxide has a number of significant drawbacks. Thus, the energy-gap width of titanium dioxide is 3.0-3.2 eV; light absorption by titanium dioxide lies in the UV region of the spectrum, that is why the efficiency of photocatalysts under visible radiation is less than $10 \%$. There is also insufficiently high quantum yield of phototransformation, which is connected with the high degree of recombination of charge carriers, low specific surface, as well as low adsorption capacity of $\mathrm{TiO}_{2}$ $[4,5]$. Therefore, it became necessary to find an alternative to $\mathrm{TiO}_{2}$. In turn, photocatalysts based on tungsten oxide $\mathrm{WO}_{3}$ are among the best materials operating under visible light [6-8]. $\mathrm{WO}_{3}$ has a energy-gap width of $2.6 \mathrm{eV}$, is stable under acidic and oxidizing conditions, has chemical stability in the $\mathrm{pH}$ range $(\mathrm{pH}<8)$, significant photocurrent conversion efficiency, is resistant to corrosion processes, stable in various electrolytes and, most importantly, is considered harmless.

The analysis of works of similar studies revealed that mainly the studies of photocatalytic activity of tungsten trioxide are aimed at studying and developing methods of chemical synthesis of nanodispersed tungsten oxide in forms suitable for its subsequent use [9-13]. Meanwhile, there are no studies on the photocatalytic activity of tungsten trioxide obtained by recycling tungsten carbide waste. Studying the properties of tungsten trioxide will enable the development of more effective photocatalysts for the oxidation of organic pollutants to $\mathrm{CO}_{2}$ and $\mathrm{H}_{2} \mathrm{O}$.

\section{Methodology for investigating the photocatalytic activity of tungsten oxide nanopowder}

The decomposition process of methylene blue was studied by spectrophotometry and by the color change of the solution during the photocatalytic reaction. A sample of nanopowder weighing 0.2 $\mathrm{g}$ was placed in a round flat-bottomed flask and $200 \mathrm{ml}$ of methylene blue solution (solution concentrations of $9,13,15$, and $20 \mathrm{mg} / \mathrm{L}$ ) was added.

The solution was irradiated with a standard white LED lamp with a temperature of $4000 \mathrm{~K}$ in the wavelength range of $380 \ldots 780 \mathrm{~nm}$. Stirring was performed on a magnetic stirrer "Ritm-01" 
with a speed of rotation of the armature of $500 \mathrm{rpm}$. Centrifugation was performed on a "CentriFuga 80-1" centrifuge, at $3000 \mathrm{rpm}$. UV-vis spectra were recorded for aqueous solutions on spectrophotometer "Expert 003" in quartz cuvettes with a width of $1 \mathrm{~cm}$.

Sampling was performed every 2 hours to control the course of the photochemical reaction and to observe changes in the concentration of the pollutant in the solution, and $10 \mathrm{ml}$ of the sample was centrifuged for $15 \mathrm{~min}$ at $3000 \mathrm{rpm}$, separating the sediment. After that the solution was decanted and the dye concentration was evaluated on a photometer "Expert 003 " by the degree of discoloration of the solution. After analysis, the sample was returned to the original solution.

From the works $[14,15]$ it was revealed that the decomposition of solutions of methylene blue passes through the kinetics of the first order, which is expressed by the equation:

$$
\ln \left(\mathrm{C}_{0} / \mathrm{C}\right)=\mathrm{k}_{\mathrm{app}} \cdot \mathrm{t}
$$

Where $\mathrm{C}_{0}$ - is the initial concentration of the methylene blue solution;

$\mathrm{C}$ - concentration at a given time;

$\mathrm{k}_{\mathrm{app}}$ - decomposition rate constant.

To determine the rate constant of decomposition in our case, we plotted $\ln \left(\mathrm{C}_{0} / \mathrm{C}\right)$ as a function of time shown in Figure 1.

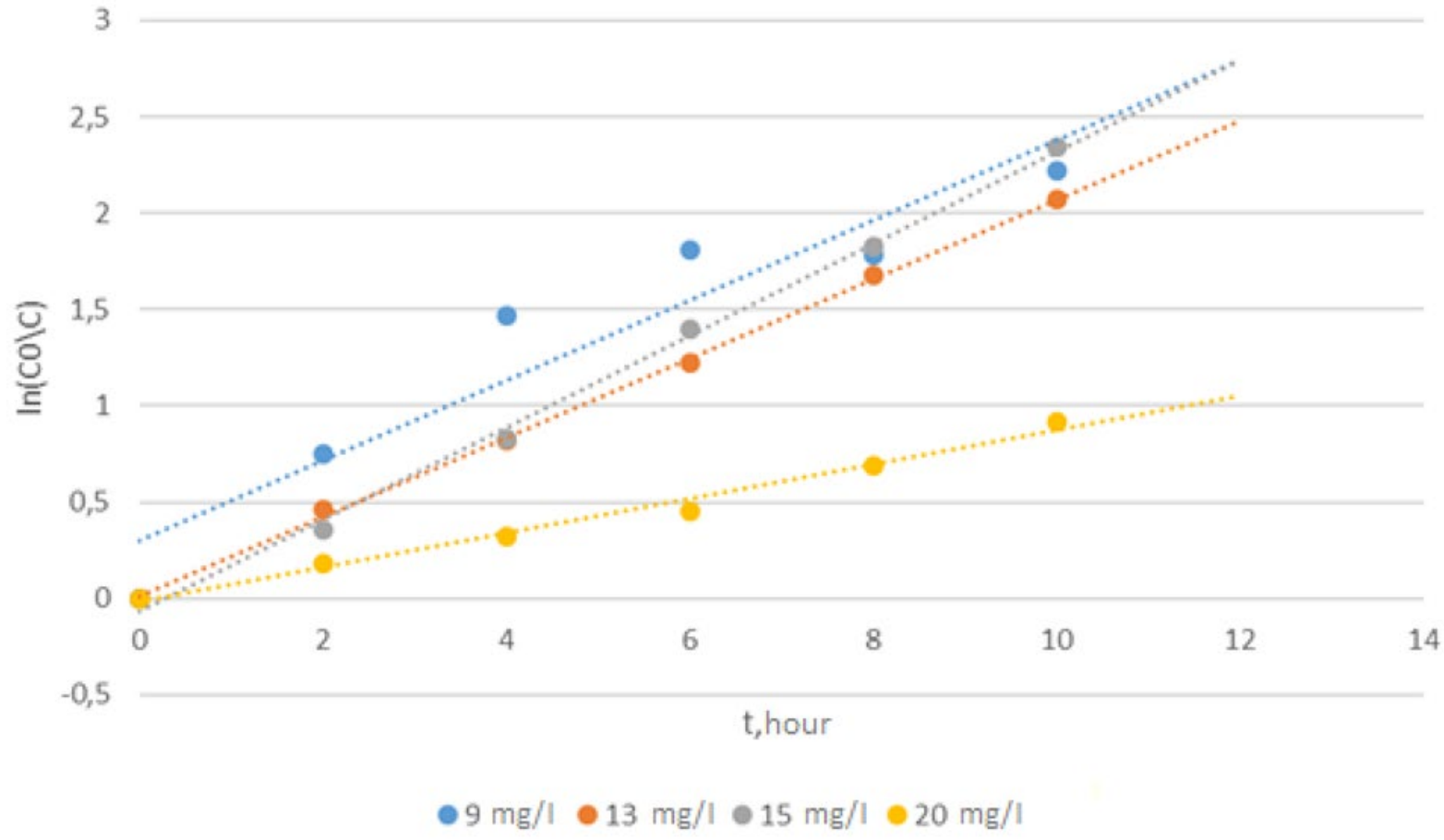

Fig. 1. - Rate constant of decomposition at different concentrations of methylene blue

The linear dependence of the data between $\ln \left(\mathrm{C}_{0} / \mathrm{C}\right)$ and irradiation time confirmed the applicability of this model with correlation coefficient values of $0.9489 ; 0.9995 ; 0.9984$ and 0.9931. The decomposition rate constant $\left(\mathrm{k}_{\mathrm{app}}\right)$ obtained from the slope of the plot was $3.7 \times 103$ min-1, $3.45 \times 103$ min-1, $3.9 \times 103 \mathrm{~min}-1$, and $1.53 \times 103 \mathrm{~min}-1$.for concentrations of $9,13,15$, 
and $20 \mathrm{mg} / \mathrm{L}$, respectively. Thus, the higher the $\mathrm{k}_{\mathrm{app}}$, the shorter the time required for complete decomposition of methylene blue.

The presented data show that the photocatalytic activity of tungsten oxide is most effective for the concentration of methylene blue $15 \mathrm{mg} / \mathrm{l}$.

\section{Study of photocatalytic activity at temperature exposure}

We also studied the effect of temperature on the photocatalytic activity of tungsten oxide. For this purpose, aqueous solutions of methylene blue with a concentration of $15 \mathrm{mg} / \mathrm{L}$ were heated with a magnetic stirrer at constant stirring for 10 hours at temperatures 15,25 , and $30{ }^{\circ} \mathrm{C}$. Methylene blue concentration drop was measured after 10 hours.

The results of the calculations are shown in figure 2.

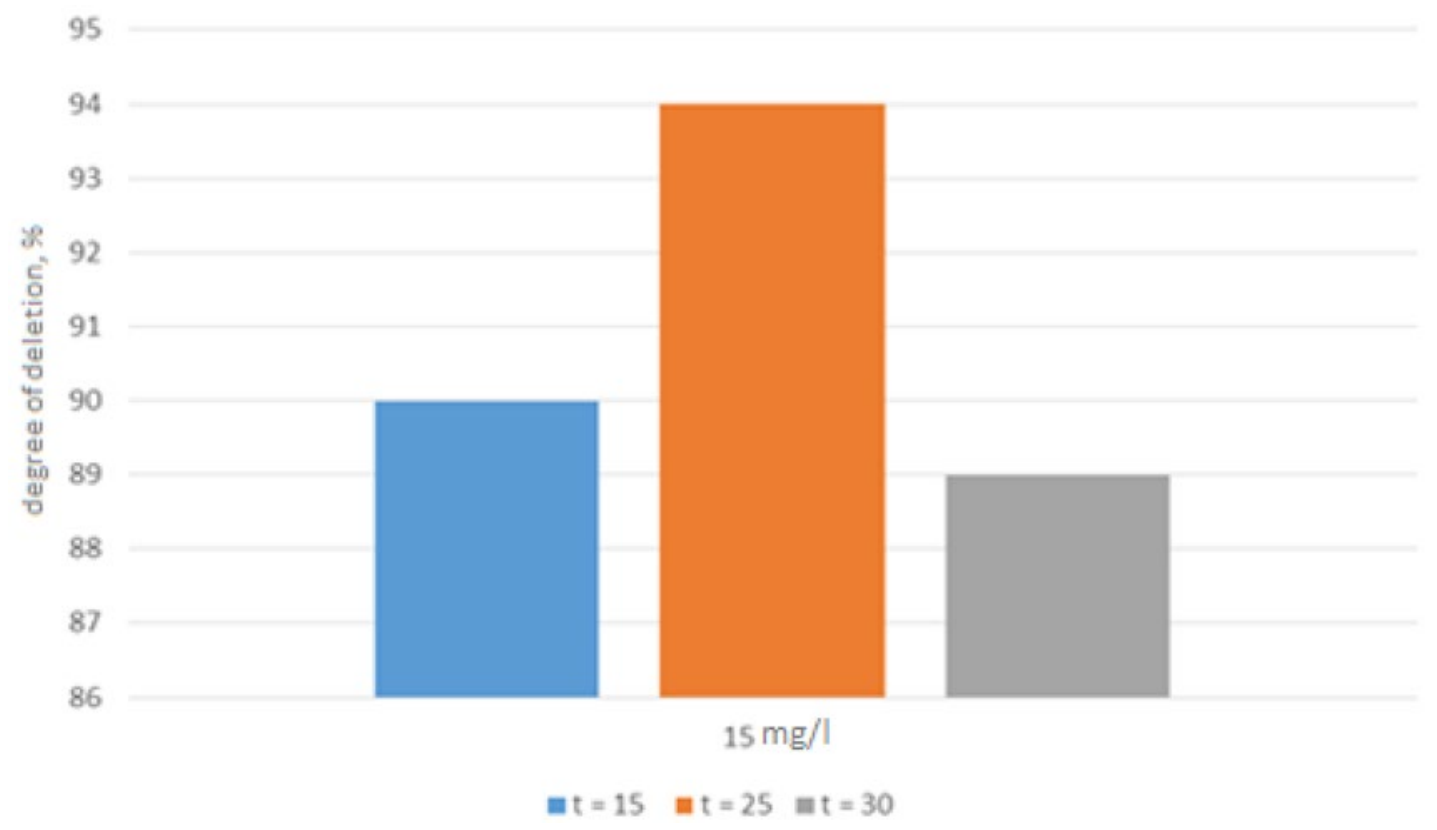

Fig. 2. Photocatalytic activity data for different temperatures

After 10 hours of continuous process, the dye spectrum value decreased by 90,94 and $89 \%$. From the analysis of the data obtained, we can conclude that the decomposition process of methylene blue is most effective at a temperature of $25{ }^{\circ} \mathrm{C}$.

\section{Conclusions}

It was found that tungsten oxide has maximum photocatalytic activity under the action of visible light in the photodegradation of the dye methylene blue at a concentration of $15 \mathrm{mg} / \mathrm{l}$. It was shown that the temperature optimum of the process is $25{ }^{\circ} \mathrm{C}$.

\section{References}

[1] Carbuloni, C.F., Savoia, J.E., Santos, J.S.P., Pereira, C.A.A., Marques, R.G., Ribeiro, V.A.S.,Ferrari, A.M. Degradation of metformin in water by $\mathrm{TiO}_{2}-\mathrm{ZrO}_{2}$ photocatalysis. J. Environ. Manag. 262 (2020) 110347.https://doi.org/10.1016/j.jenvman.2020.110347

[2] Qiong Sun, Songhao Wu, Kaijing Li, Bing Han, Yingjie Chen, Beili Pang, Liyan Yu, L. Dong The favourable synergistic operation of photocatalysis and catalytic oxygen reduction reaction by a novel heterogeneous $\mathrm{CoFe}_{2} \mathrm{O}_{4}-\mathrm{TiO}_{2}$ nanocomposite Appl. Surf. Sci., 516 (2020)146142. https://doi.org/10.1016/j.apsusc.2020.146142 
[3] E. Mrotek, S. Dudziak, I. Malinowska, D. Pelczarski, Z. Ryzynska, A. Zielinska-Jurek Improved degradation of etodolac in the presence of core-shell $\mathrm{ZnFe}_{2} \mathrm{O}_{4} / \mathrm{SiO}_{2} / \mathrm{TiO}_{2}$ magnetic photocatalyst Sci. Total Environ., 724 (2020) 138167. https://doi.org/10.1016/j.scitotenv.2020.138167

[4] H. Moradi, A. Eshaghi, S.R. Hosseini, K. Ghani Fabrication of Fe-doped TiO2 nanoparticles and investigation of photocatalytic decolorization of reactive red 198 under visible light irradiation Ultrason. Sonochem., 32 (2016) 314-319. https://doi.org/10.1016/j.ultsonch.2016.03.025

[5] J.G.S.M.J. Valero-Romero, L. Oar-Arteta, L. van Koppen, D.Y. Osadchii, J. Gascon, F. Kapteijn Photocatalytic properties of $\mathrm{TiO}_{2}$ and $\mathrm{Fe}$-doped $\mathrm{TiO}_{2}$ prepared by metal organic framework-mediated synthesis Chem. Eng. J., 360 (2019) 75-88. https://doi.org/10.1016/j.cej.2018.11.132

[6] C. Yu, F. Chen, Z. Liu, K. Yang, H. Ji, D. Li, W. Xie, S. Li Facile synthesis of a robust visible-light-driven $\mathrm{AgCl} / \mathrm{WO}_{3}$ composite microrod photocatalyst J. Alloys Compd., 809 (2019) 151844-151854. https://doi.org/10.1016/j.jallcom.2019.151844

[7] Cherkashina N.I., Pavlenko V.I., Yastrebinsky R.N. Phase transitions and change of electrophysical properties of WO3 in the temperature range 83-673 K // Proceedings of higher educational institutions. Physics. T. 62. № 5 (737) (2019)126-131. https://doi.org/10.1007/s11182-019-01789-7

[8] Pavlenko V.I., Bondarenko G.G., Cherkashina N.I. Physical and mechanical characteristics of the composite based on polyimide matrix filled with tungsten oxide, Perspective Materials № 7 ( 2019) 15-25. https://doi.org/10.30791/1028-978X-2019-7-15-25

[9] S. Adhikari, D. Sarkar High efficient electrochromic $\mathrm{WO}_{3}$ nanofibers Electrochim. Acta., 138 (2014) 115-123. https://doi.org/10.1016/j.electacta.2014.06.062

[10] J.C. Murillo-Sierra, A. Hernández-Ramírez, L. Hinojosa-Reyes, J.L. Guzmán-Mar A review on the development of visible light-responsive $\mathrm{WO}_{3}$-based photocatalysts for environmental applications Chem. Eng. J. Adv., 5 (2021) 100070-100091. https://doi.org/10.1016/j.ceja.2020.100070

[11] M.A.M. Khan, S. Kumar, T. Ahamad, A.N. Alhazaa Enhancement of photocatalytic and electrochemical properties of hydrothermally synthesized $\mathrm{WO}_{3}$ nanoparticles via Ag loading J. Alloys Compd., 743 (2018) 485-493. https://doi.org/10.1016/j.jallcom.2018.01.343

[12] M. Ahmadi, R. Younese, M.-J.-F. Guinel Synthesis of tungsten oxide nanoparticles using hydrothermal method at ambient pressure J. Mater. Res., 29 (2014) 1424-1430. https://doi.org/10.1557/jmr.2014.155

[13] R. Ponnusamy, A. Gangan, B. Chakraborty, C.S. Rout Tuning the pure monoclinic phase of $\mathrm{WO}_{3}$ and $\mathrm{WO}_{3}-\mathrm{Ag}$ nanostructures for non-enzymatic glucose sensing application with theoretical insight from electronic structure simulations J. Appl. Phys., 123 (2018) 024701024710. https://doi.org/10.1063/1.5010826

[14] R. Xu, M. Su, Y. Liu, Z. Chen, C. Ji, M. Yang, X. Chang, D. Chen, J. Clean. Prod. 242 (2020) 118366. https://doi.org/10.1016/j.jclepro.2019.118366

[15] Q. Wang, X. Lei, F. Pan, D. Xia, Y. Shang, W. Sun, W. Liu, Colloid. Surf. A. 555 (2018) 605-614. https://doi.org/10.1016/j.colsurfa.2018.07.016 